\title{
Validation of the Leicester Cough Questionnaire in non-cystic fibrosis bronchiectasis
}

\author{
M.P. Murray, K. Turnbull, S. MacQuarrie, J.L. Pentland and A.T. Hill
}

ABSTRACT: Health-related quality of life is a potentially important marker for evaluating existing and new therapies in bronchiectasis. The Leicester Cough Questionnaire (LCQ) is a symptom specific questionnaire designed to assess the impact of cough severity, a major symptom of bronchiectasis. This study aimed to validate the LCQ in bronchiectasis.

The validity, responsiveness and reliability of the LCQ were assessed as follows: ability to discriminate severe and mild disease; change in score following antibiotic treatment for exacerbations; repeatability over a 6-month period in stable disease; and comparison with the St George's Respiratory Questionnaire (SGRQ).

In total, 120 patients (51 with severe disease, 29 with moderate disease and 40 with mild disease) completed the LCQ and SGRQ. The area under the receiver-operator curve was good for both severe and mild disease $(0.84$ and 0.80 respectively, $p<0.0001)$. Following 2 weeks' antibiotic treatment, the median LCQ score (interquartile range) improved from 11.3 (9.3-13.7) to $17.8(15-18.8)(p<0.0001)$. The $L C Q$ score was repeatable over 6 months in stable disease (intraclass correlation coefficient of $0.96(95 \% \mathrm{Cl} 0.93-0.97), p<0.0001)$. Correlation between the LCQ and SGRQ scores was -0.7 in both stable disease and exacerbations $(p<0.0001)$.

The LCQ can discriminate disease severity, is responsive to change and is reliable for use in non-cystic fibrosis bronchiectasis.

KEYWORDS: Bronchiectasis, cough, health-related quality of life

B ronchiectasis, first described by LAENNEC [1] in 1819, is a chronic debilitating condition, with abnormal permanent dilatation of the airways causing impaired mucus clearance, chronic bacterial infection and persistent bronchial inflammation. Patients suffer from daily cough, excess sputum production and frequent exacerbations. They may also report breathlessness, wheeze and fatigue. Such debilitating symptoms impact on patients' health-related quality of life (HRQoL) [2, 3]. Improving patients' HRQoL is a major goal of disease management.

Assessing HRQoL allows the clinician to directly quantify the effect of disease on patients' daily life and is recognised as an important tool in the evaluation of both existing and new therapies [4, 5]. In chronic conditions, questionnaires targeting symptoms specific to the condition, exploring their physical effects, the subsequent restrictions in daily life and effect on mood are useful and relevant [6, 7]. Such questionnaires provide a unique integration of physical and psychosocial morbidity, unlike other clinical parameters.
The only validated questionnaire used in noncystic fibrosis bronchiectasis is the St George's Respiratory Questionnaire (SGRQ) [3]. This was originally designed for use in chronic obstructive pulmonary disease (COPD) and assesses multiple symptoms, including dyspnoea, wheeze, cough and sputum production [8]. A symptom specific questionnaire focussing purely on cough and sputum expectoration, the major symptoms of bronchiectasis, would be an ideal measure of HRQoL for bronchiectasis patients.

The Leicester Cough Questionnaire (LCQ) is a HRQoL questionnaire validated for assessing chronic cough [9]. It is a 19-item, self-completed questionnaire exploring the impact of cough severity across three domains: physical (eight items), psychological (seven items) and social (four items). The total severity score ranges from 3 to 21 , with a lower score indicating greater impairment of health status due to cough. It assesses the impact of symptoms over the preceding 2 weeks and its potential has recently been explored in a range of conditions including

\section{AFFILIATIONS}

Dept of Respiratory Medicine, Royal Infirmary of Edinburgh, Edinburgh, UK.

\section{CORRESPONDENCE}

M.P. Murray

Royal Infirmary of Edinburgh, Department of Respiratory Medicine 51 Little France Crescent

Old Dalkeith Road

Edinburgh EH16 4SA UK E-mail: maevemurray@hotmail.com Received: Oct 242008 Accepted after revision: Jan 112009 First published online: Feb 052009 
COPD, asthma and bronchiectasis [10]. However, no studies have focussed solely on non-cystic fibrosis bronchiectasis and it has not been validated for use in bronchiectasis.

The LCQ offers a pertinent, timely and useful clinical tool for bronchiectasis. It focuses purely on the impact of cough severity on HRQoL, unlike other questionnaires that encompass multiple respiratory symptoms. Cough is the dominant symptom of bronchiectasis, present in up to $98 \%$ of patients and measuring its impact on HRQoL is critical for both disease assessment and targeted management [11]. Additionally, the LCQ is concise, consisting of only 19 items, offering greater patient acceptance than lengthier questionnaires.

The aim of this study was to validate the utility of the LCQ as an assessment tool in non-cystic fibrosis bronchiectasis.

\section{METHODS}

This was a 2-yr prospective cohort study (2006-2008) in noncystic fibrosis bronchiectasis approved by Lothian Research Ethics Committee (Lothian, UK). To validate the LCQ, we explored its validity, responsiveness and reliability. For validity, we assessed the sensitivity, specificity, positive and negative predictive values (PPV and NPV, respectively), likelihood ratios, and post-test odds, as well as the area under the receiver-operator curve in predicting both severe and mild bronchiectasis. In addition, we assessed the correlation of the LCQ with the SGRQ in stable disease and in exacerbations. For responsiveness, we assessed the change in LCQ score following antibiotic treatment for exacerbations. For reliability, we assessed change in LCQ score over a 6-month period when clinically stable.

\section{Questionnaires}

The LCQ is a 19-item, self-completed, quality-of-life measure of chronic cough. It has three domains: physical (eight items), psychological (seven items) and social (four items) [9]. The total severity score ranges 3-21, with a lower score indicating greater impairment of health status due to cough. It assesses the impact of symptoms over the preceding 2 weeks.

The SGRQ is a 50 -item, self-administered HRQoL questionnaire consisting of three components: symptoms (eight items), activity (16 items) and impacts (26 items). It has previously been validated to reflect impaired HRQoL in bronchiectasis patients [3]. The total score ranges $0-100$, with a higher score indicating a poorer HRQoL. It assesses the impact of symptoms over the preceding 4 weeks.

\section{Validity}

All patients with an established diagnosis of bronchiectasis based on both clinical history and computed tomography (CT) of the chest and who regularly attended the regional bronchiectasis clinic were invited to participate in the study.

Patients were recruited if they were clinically stable (defined as no requirement for antibiotics in the preceding 4 weeks) and had no exclusion criteria. All patients completed the LCQ and the SGRQ $24 \mathrm{~h}$ prior to routine outpatient clinic attendance.

Exclusion criteria were: long-term antibiotic therapy (oral or nebulised); current smokers and ex-smokers of $\leqslant 2$ yrs; coexisting COPD with forced expiratory volume in $1 \mathrm{~s}$ (FEV1)
$<60 \%$ predicted; a primary diagnosis of asthma; cystic fibrosis; active sarcoidosis, active allergic bronchopulmonary aspergillosis; or active tuberculosis.

To predict severe and mild bronchiectasis, the following criteria were used. Severe bronchiectasis was defined as meeting all of the following clinical, microbiological and radiological criteria: expectorating purulent sputum when stable assessed using a standardised sputum colour chart [12]; chronic sputum colonisation (defined as pathogenic bacteria cultured in at least two sputum samples when clinically stable in the preceding 12 months) and at least three lobes affected on CT scan, with evidence of varicose or cystic bronchial dilatation in at least one lobe (for the purposes of this study, the lingula was considered as a separate lobe). Mild bronchiectasis was defined as meeting all of the following clinical, microbiological and radiological criteria: expectorating mucoid or no sputum when clinically stable assessed using a standardised sputum colour chart [12]; evidence of cylindrical bronchiectasis on CT scan in three or fewer lobes; and no evidence of chronic sputum colonisation. All other patients were classified as having moderate bronchiectasis.

\section{Responsiveness}

Patients with exacerbations, defined as increasing cough, increasing sputum volume, worsening sputum purulence and a need for intravenous antibiotic therapy, were recruited. Both LCQ and SGRQ questionnaires were filled in immediately prior to commencing antibiotic treatment and 1 week following completion of treatment. Both questionnaires were adapted for the end of exacerbation assessment to ask about symptoms in the preceding week.

For patients who had experienced more than one exacerbation, only the first exacerbation was used. All patients received 14 days of i.v. antibiotics treatment based on individual sputum bacteriology.

\section{Reliability}

The first 75 patients with clinically stable bronchiectasis (irrespective of disease severity) and who had had no changes made to therapy in the 6 months following entry to the study, were invited to repeat the LCQ 6 months after completion of the initial questionnaire.

\section{STATISTICS}

The utility of the LCQ in indicating severe and mild disease was assessed using sensitivity and specificity, PPV, NPV, likelihood ratio for a positive test and likelihood ratio for a negative test and the post-test odds as well as the area under the receiver-operator curve (AUC). The Kruskal-Wallis test compared severe bronchiectasis with moderate and mild bronchiectasis. The Wilcoxon test was used to compare scores at the start and end of exacerbations. The Spearman's rank correlation was used to assess correlation of the LCQ with SGRQ. For assessment of reliability over time, the intraclass correlation coefficient (ICC) was measured. A Bland-Altman plot was used to display repeatability over time [13]. Statistical analysis was performed using SPSS for Windows, Version 16 (SPSS Inc., Chicago, IL, USA) and Graphpad Prism Version 5.0 (GraphPad Software, San Diego, CA, USA). For interpretation of AUC values the following is widely accepted: AUC 0.50-0.59 


\section{TABLE 1 Patient characteristics}

\begin{tabular}{|c|c|c|c|c|}
\hline \multirow[t]{2}{*}{ Characteristic } & \multicolumn{3}{|c|}{ Bronchiectasis } & \multirow[t]{2}{*}{$p$-value } \\
\hline & Severe & Moderate & Mild & \\
\hline Subjects $\mathrm{n}$ & 51 & 29 & 40 & \\
\hline Male & $25(49)$ & $10(34.5)$ & $16(40)$ & 0.4 \\
\hline Age yrs & $68(60-74)$ & $69.5(56.2-77.2)$ & $67(59-76.7)$ & 0.9 \\
\hline FEV 1 L & $1.55(1.30-2.15)$ & $1.8(1.34-2.47)$ & $2.3(1.9-2.7)$ & 0.002 \\
\hline FEV $1 \%$ pred & $69(47-86)$ & $78.2(69.7-89.6)$ & $82.5(71.5-96)$ & 0.006 \\
\hline FVC L & $2.45(1.80-3.20)$ & $2.55(2-3.19)$ & $3.06(2.5-3.5)$ & 0.04 \\
\hline FVC \% pred & $77(61-93)$ & $85.9(85-100)$ & $90(78.5-106)$ & 0.01 \\
\hline $\mathrm{FEV}_{1 / \mathrm{FVC}}$ ratio & $0.68(0.58-0.77)$ & $0.76(0.65-0.85)$ & $0.74(0.66-0.82)$ & 0.01 \\
\hline $\mathrm{FEV}_{1 / \mathrm{FVC}} \%$ pred & 89 (73-97) & $94(85-105)$ & $94.9(86-102)$ & 0.09 \\
\hline Lobes affected & $5(4-6)$ & $4(1.5-5)$ & $2(1-3)$ & $<0.0001$ \\
\hline Outpatient exacerbations in the preceding 12 months & $3(1.75-5.25)$ & $1(0-3)$ & $0(0-1.25)$ & $<0.0001$ \\
\hline
\end{tabular}

shows no value of test; $0.6-0.69$ shows poor discrimination value; $0.70-0.79$ shows moderate discriminatory value; $0.80-0.89$ shows good discriminatory value; and 0.9-1.0 shows excellent discriminatory value [14]. A 2-tailed p-value of $<0.05$ was statistically significant. Data are presented as median (interquartile range).

\section{RESULTS}

Validity: the LCQ as a discriminant of severe bronchiectasis In total, 120 patients were eligible for inclusion in the final analysis. Of these, 51 were classified as having severe bronchiectasis, 29 as moderate and 40 as mild. Table 1 details

\section{TABLE 2 Aetiology of bronchiectasis}

\begin{tabular}{lccc} 
Aetiology & \multicolumn{3}{c}{ Bronchiectasis } \\
\cline { 2 - 4 } & Severe & Moderate & Mild \\
\hline Subjects $\mathbf{n}$ & 51 & 29 & 40 \\
Idiopathic & 14 & 45 & 38 \\
Post-infective & 47 & 35 & 43 \\
Post-pulmonary tuberculosis & 12 & 17 & 7 \\
Allergic bronchopulmonary & 17 & 3 & 5 \\
$\quad$ aspergillosis & 2 & & \\
Inflammatory bowel disease & 2 & 0 & 0 \\
IgG subclass deficiency & 2 & 0 & 5 \\
Ciliary dyskinesia & 4 & 0 & 2 \\
Sarcoidosis & & & 0 \\
\hline
\end{tabular}

Data are presented as \%, unless otherwise stated. Ig: immunoglobulin. baseline characteristics for each group and table 2 provides details of the aetiology of the bronchiectasis for each group.

In predicting severe bronchiectasis, the LCQ performed well, with an AUC of $0.84(0.80-0.88, p<0.0001$; fig. 1). The lower the LCQ score the greater the likelihood ratio for severe disease (table 3). In predicting mild bronchiectasis, the LCQ performed well, with an AUC of 0.80 (0.76-0.84, p $<0.0001$; fig. 1). The higher the LCQ score the greater the likelihood ratio for mild disease (table 4).

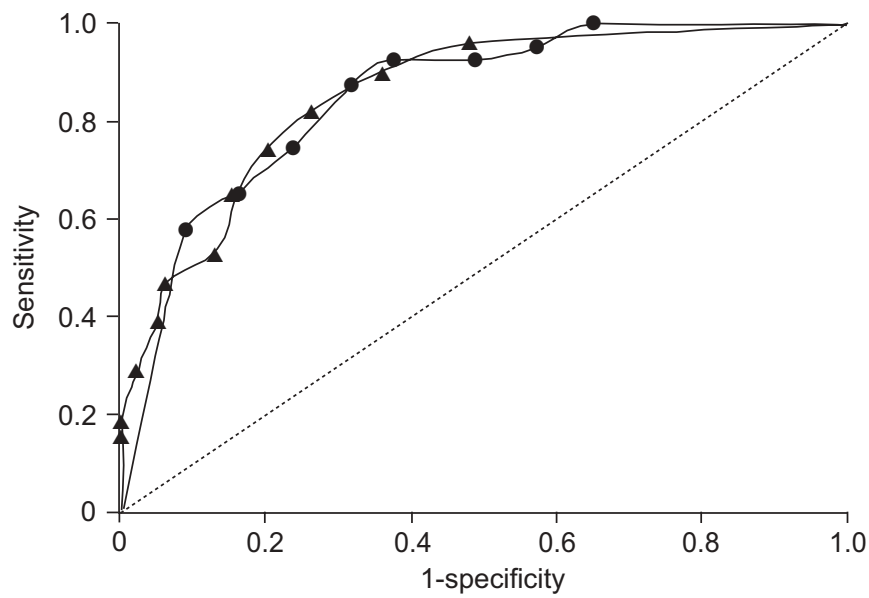

FIGURE 1. Validity of the Leicester Cough Questionnaire (LCQ). Receiveroperator curve in severe bronchiectasis $(\mathbf{\Lambda}$; area under the curve $(A \cup C)=0.84)$ and in mild bronchiectasis $(-$ AUC $=0.80$ ). Total LCQ Score cut-off values used: $3-5$ 6-8; 9-11; 12-14; 15-17; 18-21. Straight line AUC (----)=0.5. 
TABLE 3 Utility of the Leicester Cough Questionnaire (LCQ) predicting severe disease

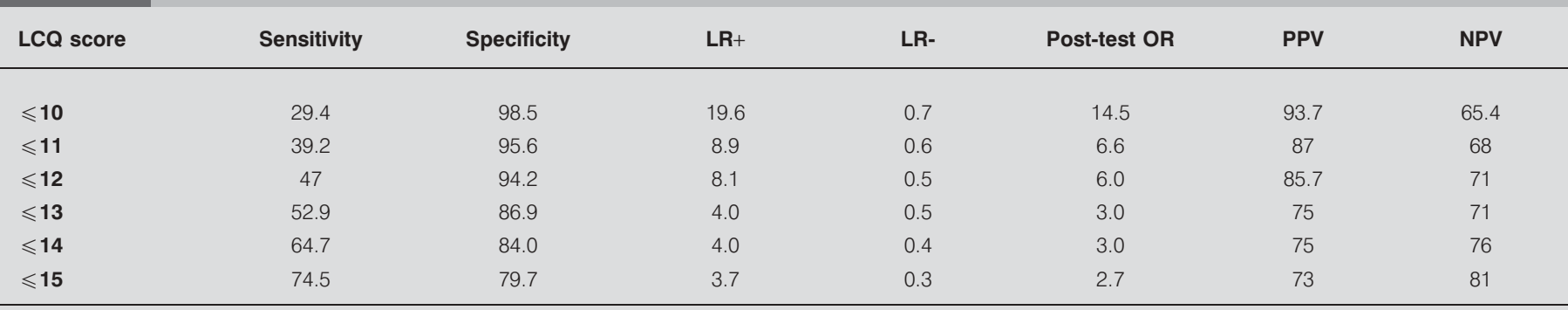

Prevalence was $42.5 \%$ and pre-test odds ratio (OR) was 0.74 . LR+: likelihood ratio for a positive test; LR-: likelihood ratio for a negative test; PPV: positive predictive value NPV: negative predictive value.

TABLE 4 Utility of the Leicester Cough Questionnaire (LCQ) predicting mild disease

\begin{tabular}{|c|c|c|c|c|c|c|c|}
\hline LCQ score & Sensitivity & Specificity & LR+ & LR- & Post-Test OR & PPV & NPV \\
\hline$\geqslant 20$ & 57.5 & 91.2 & 6.5 & 0.5 & 3.3 & 76.7 & 81.1 \\
\hline$\geqslant 18$ & 75 & 76.2 & 3.15 & 0.3 & 1.6 & 61.2 & 85.9 \\
\hline$\geqslant 17$ & 87.5 & 68.7 & 2.8 & 0.18 & 1.4 & 58.3 & 91.7 \\
\hline
\end{tabular}

Prevalence was $33.33 \%$ and pre-test odds ratio (OR) was 0.5 . LR+: likelihood ratio for a positive test; LR-: likelihood ratio for a negative test; PPV: positive predictive value; NPV: negative predictive value.

The total and individual domain scores (physical, psychological and social) were significantly lower in those with severe disease compared with mild and moderate disease (table 5).

The LCQ had a significant inverse correlation with the SGRQ in stable disease. The Spearman rank correlation coefficient was -0.7 , (95\% CI $-0.58--0.78 ; \mathrm{p}<0.0001$; fig. 2a).

\section{Responsiveness: the $L C Q$ at the start and end of antibiotic treatment}

In total, 32 exacerbations met the study criteria and were eligible for inclusion in the final analysis. Of these, 19 were due to Pseudomonas aeruginosa and 13 were due to other potentially pathogenic microorganisms (three Haemophilus influenzae, three Streptococcus pneumoniae, two methicillin-resistant Staphylococcus aureus, two Moraxella cattarhalis, one S. aureus, one Escherichia coli and one Serratia spp.). There was a significant improvement

\begin{tabular}{lccc} 
TABLE 5 & Leicester Cough Questionnaire scores \\
\hline Domain & $\begin{array}{c}\text { Severe } \\
\text { bronchiectasis }^{\#}\end{array}$ & $\begin{array}{c}\text { Moderate } \\
\text { bronchiectasis }^{\text {* }}\end{array}$ & $\begin{array}{c}\text { Mild } \\
\text { bronchiectasis }^{+}\end{array}$ \\
\hline Physical & $4.4(3.4-5)$ & $5.5(4.4-6.2)$ & $6.1(5.2-6.7)^{\S}$ \\
Psychological & $4.4(3.3-5.3)$ & $5.9(4.8-6.8)$ & $6.8(5.7-7.0)^{\S}$ \\
Social & $4.5(3.5-6.0)$ & $6.0(5.0-6.9)$ & $6.7(6.0-7.0)^{\S}$ \\
Total & $13.6(10.4-16.2)$ & $17.8(14.02-19.9)$ & $19.3(16.9-20.6)^{\S}$ \\
\hline
\end{tabular}

Data are presented as median (interquartile range). ${ }^{*}: n=51 ; \bullet: 29 ;{ }^{+}: 40$. s. $p<0.0001$ $(\mathrm{p}<0.0001)$ in both the individual domain and total scores between the start and end of treatment. The actual domain scores at the start and end of treatment, respectively, were: physical domain (3.5 (2.9-4) and 5.4 (4.4-5.7)); psychological domain (3.8 (2.9-5) and $6.1(4.8-6.7))$; and social domain (4.0 $(2.7-4.7)$ and $6.0(5.2-6.5))$. The total LCQ score at the start of treatment was $11.3(9.3-13.8)$ and at the end of treatment was 17.8 (14.2-18.8). The changes for each patient are shown in figure 3a. The LCQ had a significant inverse correlation with the SGRQ (Spearman rank correlation coefficient of -0.69 (95\% CI $-0.53--0.81 ; p<0.0001$; fig. $2 b)$.

\section{Reliability: the repeatability of the LCQ in stable bronchiectasis over a 6-month period}

In total, $67(89.3 \%)$ patients repeated the LCQ 6 months after completion of the initial questionnaire. The ICC for the total LCQ score was 0.96 (95\% CI 0.93-0.97; $\mathrm{p}<0.0001)$. The mean \pm SD difference between the total scores was $-0.23 \pm 1.1$. A Bland-Altman plot of the difference between repeat total scores for the LCQ is shown in figure $3 b$.

\section{DISCUSSION}

This study establishes the validity, responsiveness and reliability of the LCQ in non-cystic fibrosis bronchiectasis. The validation of this HRQoL questionnaire, which explores the impact of cough severity, is relevant to bronchiectasis and is likely to be of value in the evaluation of both existing and new therapeutic agents.

HRQoL questionnaires are well established useful measures of the direct impact of chronic respiratory disease on patients' daily lives [15-17]. In chronic conditions, it has been increasingly 


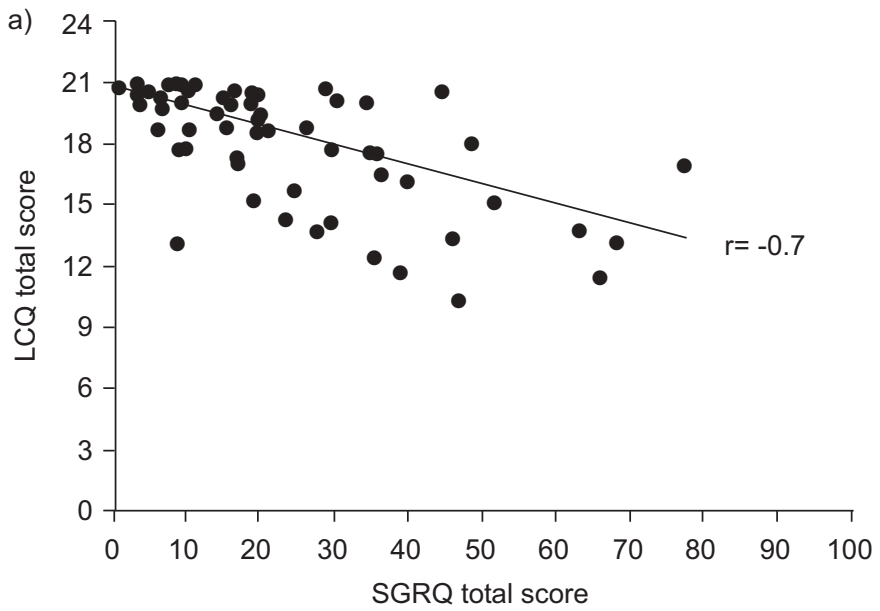

b)

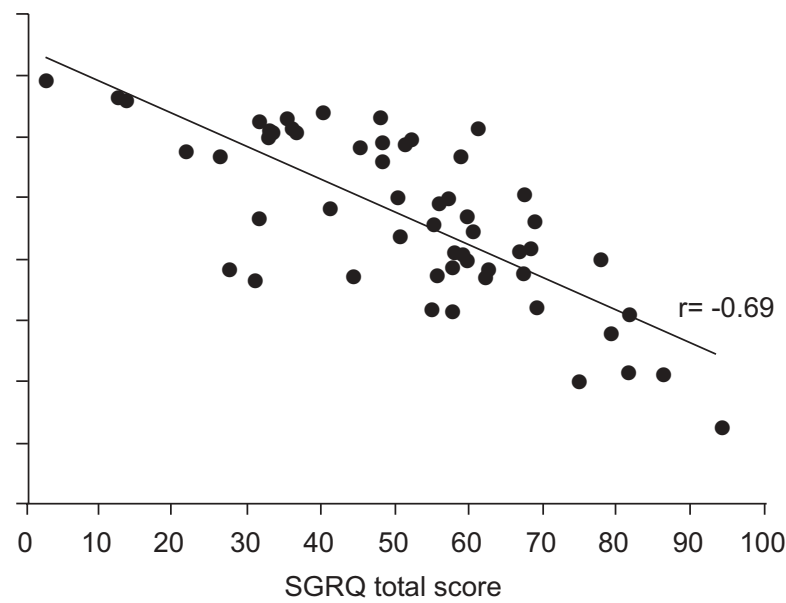

FIGURE 2. Validity of the Leicester Cough Questionnaire (LCQ). Correlation of the LCQ total score with the St George's Respiratory Questionnaire (SGRQ) total score in a) stable bronchiectasis and b) exacerbations.

recognised over the past decade that improving quality of life is important [4]. Achieving an improvement in HRQoL is a major treatment target in bronchiectasis. However, to date, there is a distinct paucity of validated questionnaires available to assess HRQoL in non-cystic fibrosis bronchiectasis. There is no questionnaire that has been specifically developed for the disease and, to our knowledge, there is only one validated questionnaire at present, the SGRQ, which was originally designed and intended to assess obstructive airways disease [3].

Cough is a cardinal symptom of bronchiectasis and, as such, may be most likely to impact on HRQoL. A questionnaire that evaluates the effect of perceived cough severity is highly relevant. The LCQ is a symptom-specific questionnaire assessing cough and was selected for validation as it focuses solely on the dominant symptom of bronchiectasis and, additionally, its potential utility in bronchiectasis has been highlighted in previous studies [10, 18]. TORREGO et al. [18] explored the sensitivity of the cough reflex in bronchiectasis using inhalation of incremental doses of capsaicin and found that capsaicin sensitivity correlated positively with the total LCQ score $(r=0.64, p=0.005)$. More recently, since the completion of this study, POLLEY et al. [10] have compared the LCQ and the Cough Quality of Life Questionnaire together with a generic health status questionnaire (the EUroQol) in different chronic respiratory diseases, including bronchiectasis, and suggested the LCQ may be able to provide useful information about the impact of cough $[19,20]$. The LCQ itself is more concise than other HRQoL questionnaires assessing cough which may imply greater patient acceptance of the questionnaire [9].

The three key concepts required to ensure a questionnaire is valid for a disease are: validity, responsiveness and reliability. Our study included all patients with an established diagnosis of bronchiectasis attending a specialist clinic. We then selected patients at different ends of the spectrum, mild and severe bronchiectasis, to establish whether the LCQ could demonstrate
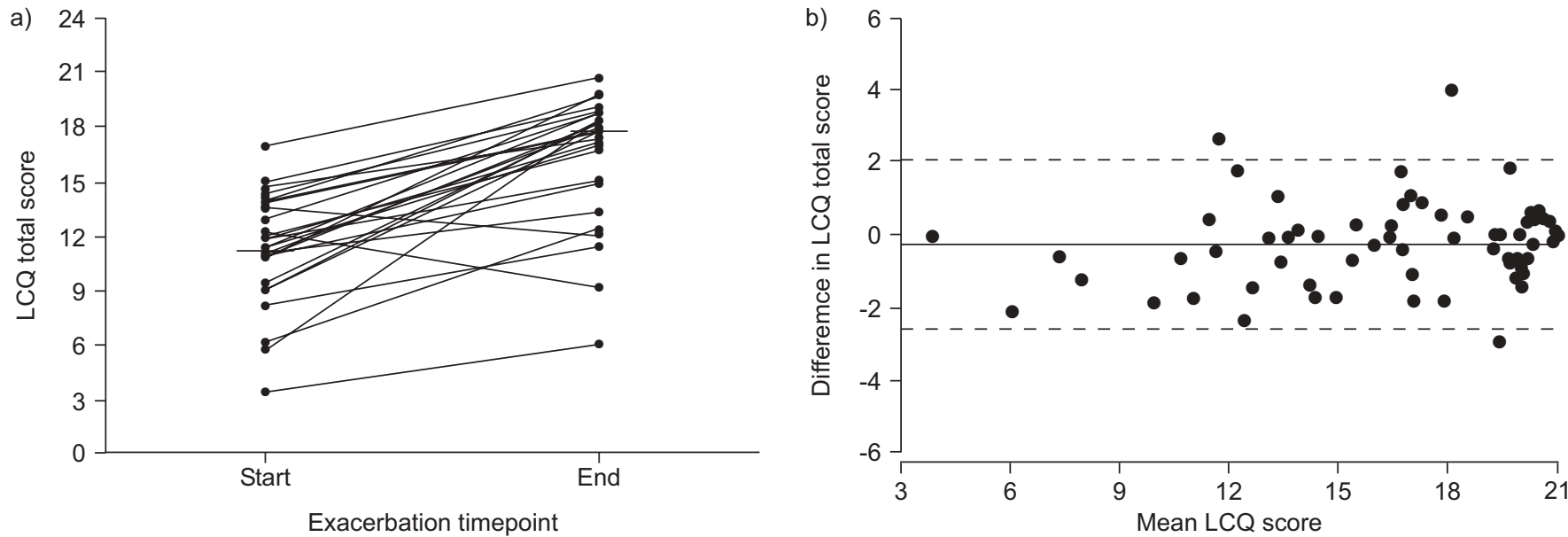

FIGURE 3. a) Responsiveness of the Leicester Cough Questionnaire (LCQ). Total score before and after 2-weeks' intravenous antibiotic treatment in 32 exacerbations.

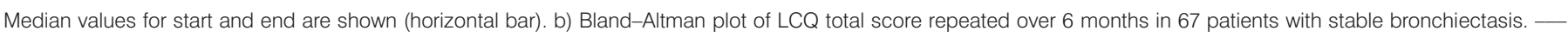
mean difference between the two scores $(-0.23)$; -----: 95\% limits of agreement (-2.6-2.1). 
convergent validity using selected relevant markers of mild and severe disease, respectively. There are no previously established criteria defining disease severity in bronchiectasis. The criteria we used to define mild and severe disease were based on our clinical experience of non-cystic fibrosis bronchiectasis and incorporate the major features of the disease, including radiological severity, microbiological history and clinical symptoms. Aspects of these key features have, in previous studies, been independently associated with HRQoL, WILSON et al. [21] explored whether sputum bacteriology affected HRQoL and found that patients colonised with $P$. aeruginosa had a significantly worse SGRQ score than other patients with $H$. influenzae or no bacterial growth $(\mathrm{p}<0.05)$. MARTINEZ-GARCIA et al. [2] found in their cohort of Spanish patients with non-cystic fibrosis bronchiectasis that dyspnoea, FEV1 and sputum expectoration were associated with a worse SGRQ score. We found that the LCQ performed well for predicting both severe and mild disease. In clinical practice, however, the main value of the LCQ will be in the longitudinal assessment of disease and in the evaluation of existing and new therapies.

To our knowledge, the SGRQ is the only previously validated HRQoL questionnaire in non-cystic fibrosis bronchiectasis [3]. We therefore used this as a standard against which to assess the LCQ. We found the LCQ total score had a significant negative correlation with the total SGRQ score in both stable disease and exacerbations, further corroborating the validity of the LCQ in non-cystic fibrosis bronchiectasis. The correlation is, however, imperfect suggesting that the two questionnaires are providing information on different aspects of the impact of bronchiectasis on HRQoL.

The LCQ confers advantages over the SGRQ in bronchiectasis. The LCQ focuses on cough, the major symptom of both stable disease and exacerbations, making it highly relevant. The SGRQ assesses other clinical features in addition to cough such as dyspnoea and wheeze, symptoms which are less frequently present in bronchiectasis; a previous study found that dyspnoea was present in only $62 \%$ of patients at presentation compared with cough in $98 \%$ [11]. The LCQ is concise, with only 19 items for completion compared with 50 items contained within the SGRQ, and will be easier to complete particularly if repeated testing is required, for example, in response to exacerbations. Furthermore, the LCQ assesses symptoms over a shorter time period (2 weeks) compared with 4 weeks in the SGRQ. The validation of the LCQ in bronchiectasis in addition to the SGRQ will provide two relevant and important means of assessing the impact of daily symptoms on HRQoL. Improving HRQoL is a major aim of management in chronic disease.

There was a significant improvement across all domains and the total LCQ score in direct response to 2-weeks' i.v. antibiotic treatment for exacerbations with a median improvement in total score of 4.6 (3.2-7.2) following completion of treatment. MutALITHAs et al. [22] found a significant improvement in all domains of the LCQ following 4 weeks of outpatient-based physical therapy intervention, with total score improving from 14.2 to 17.3 ( $p<0.0001)$. There is debate regarding the minimal important difference in total LCQ score thought to be clinically relevant, with previous studies assessing the LCQ in chronic cough suggesting a change of between 1.3 and 2.7 [23, 24]. The present study did not address the minimally important difference and further studies are needed.

The reliability of the LCQ was measured by assessing its repeatability over 6 months, a period longer than the 2-week time course used in the initial development and validation of the questionnaire [9]. A period of 6 months is representative of the routine follow-up period for patients with stable non-cystic fibrosis bronchiectasis. It is expected that there is some noise in HRQoL questionnaires but in clinically stable bronchiectasis the scores were repeatable over a 6-month period with a high intraclass correlation coefficient. The mean change for clinically stable patients over 6 months was $-0.23 \pm 1.1$ units, lower than the minimal important differences found in the previous studies.

This study validates the LCQ for use in the assessment of noncystic fibrosis bronchiectasis. It is able to discriminate disease severity, is responsive to change and is reliable. It assesses one of the most pertinent symptoms of bronchiectasis and is likely to be of value in assessment of both existing and new therapies as well as providing a relevant adjunct to current clinical management. Further studies to externally validate the LCQ in bronchiectasis would be welcome.

\section{SUPPORT STATEMENT}

M.P. Murray is funded by the Chief Scientist Office, Scotland, UK.

\section{STATEMENT OF INTEREST}

None declared

\section{ACKNOWLEDGEMENTS}

We would like to thank I. Pavord (Institute for Lung Health, University of Leicester, Leicester, UK) and S. Birring (King's College Hospital, London, UK) for their kind permission to use the Leicester Cough Questionnaire and P. Jones (St George's Hospital, London, UK) for his kind permission to use the St George's Respiratory Questionnaire.

\section{REFERENCES}

1 Laennec RT. A treatise in the diseases of the chest and on mediate auscultation. 4th Edn. Translation by J. Forbes. Longman, London, 1834.

2 Martínez-García MA, Perpiñá-Tordera M, Román-Sánchez P, et al. Quality-of-life determinants in patients with clinically stable bronchiectasis. Chest 2005; 128: 739-745.

3 Wilson CB, Jones PW, O'Leary CJ, et al. Validation of the St. George's Respiratory Questionnaire in bronchiectasis. Am J Respir Crit Care Med 1997; 156: 536-541.

4 Curtis JR, Martin DP, Martin TR. Patient-assessed health outcomes in chronic lung disease: what are they, how do they help us, and where do we go from here? Am J Respir Crit Care Med 1997; 156: 1032-1039.

5 Mahler DA. How should health-related quality of life be assessed in patients with COPD? Chest 2000; 117: Suppl. 2, 54S-57S.

6 Kalpaklioglu AF, Kara T, Kurtipek E, et al. Evaluation and impact of chronic cough: comparison of specific versus generic quality-oflife questionnaires. Ann Allergy Asthma Immunol 2005; 94: 581-585.

7 Puhan MA, Guyatt GH, Goldstein R, et al. Relative responsiveness of the Chronic Respiratory Questionnaire, St. Georges Respiratory Questionnaire and four other health-related quality of life instruments for patients with chronic lung disease. Respir Med 2007; 101: 308-316.

8 Jones PW, Quirk FH, Baveystock CM, et al. A self-complete measure of health status for chronic airflow limitation. The St. George's Respiratory Questionnaire. Am Rev Respir Dis 1992; 145: 1321-1327. 
9 Birring SS, Prudon B, Carr AJ, et al. Development of a symptom specific health status measure for patients with chronic cough: Leicester Cough Questionnaire (LCQ). Thorax 2003; 58: 339-343.

10 Polley L, Yaman N, Heaney L, et al. Impact of cough across different chronic respiratory diseases: comparison of two coughspecific health-related quality of life questionnaires. Chest 2008; 134: 295-302.

11 King PT, Holdsworth SR, Freezer NJ, et al. Characterisation of the onset and presenting clinical features of adult bronchiectasis. Respir Med 2006; 100: 2183-2189.

12 Murray MP, Pentland JL, Turnbull K, et al. Sputum colour: a useful clinical tool in non-cystic fibrosis bronchiectasis. Eur Respir J 2009 (in press); DOI: 10.1183/09031936.00163208.

13 Bland JM, Altman DG. Statistical methods for assessing agreement between two methods of clinical measurement. Lancet 1986; 1 : 307-310.

14 Swets JA. Measuring the accuracy of diagnostic systems. Science 1988; 240: 1285-1293.

15 Cox CE, Donohue JF, Brown CD, et al. Health-related quality of life of persons with sarcoidosis. Chest 2004; 125: 997-1004.

16 Jones PW. Impact of lower respiratory tract infections on health status. Semin Respir Crit Care Med 2000; 21: 107-111.
17 Swigris JJ, Gould MK, Wilson SR. Health-related quality of life among patients with idiopathic pulmonary fibrosis. Chest 2005; 127: 284-294.

18 Torrego A, Haque RA, Nguyen LT, et al. Capsaicin cough sensitivity in bronchiectasis. Thorax 2006; 61: 706-709.

19 EuroQol - a new facility for the measurement of health-related quality of life. The EuroQol Group. Health Policy 1990; 16: 199-208.

20 French CT, Irwin RS, Fletcher KE, et al. Evaluation of a coughspecific quality-of-life questionnaire. Chest 2002; 121: 1123-1131.

21 Wilson CB, Jones PW, O'Leary CJ, et al. Effect of sputum bacteriology on the quality of life of patients with bronchiectasis. Eur Respir J 1997; 10: 1754-1760.

22 Mutalithas K, Watkin G, Willig B, et al. Improvement in health status following bronchopulmonary hygiene physical therapy in patients with bronchiectasis. Respir Med 2008; 102: 1140-1144.

23 Raj AA, Pavord I, Birring SS. What is the minimal important difference for the Leicester Cough Questionnaire? Thorax 2006; 61: Suppl. 2, ii57, P109.

24 Brokkaar L, Uil S, Van Den Berg JWK. Minimal clinically important difference (MCID) of the Dutch Version of the Leicester Cough Questionnaire and baseline predictors of reaching the MCID after six months. Chest 2007; 132: Suppl. 4, 468b. 